EXEMPLARIa Classica

Journal of Classical Philology

14, 2010, pp. 175-183

ISSN 1699-3225

\title{
LA GALLINA DI PETRONIO E LE ALTRE
}

\author{
Romeo Schievenin \\ Università di Padova \\ romeo.schievenin@unipd.it
}

SUMMARY

The episode of the chicken and peahen's eggs presents some technical factors that on one hand allow to delete without hesitation a gloss of the Codex Traguriensis, on the other involve interesting implications on the literary level for the interpretation of the whole episode.

KEYWORDS

Petronius, chicken, eggs.
RESUMEN

L'episodio della gallina e delle uova di pavone presenta elementi tecnici che da una parte permettono di riconoscere con certezza una glossa del Codice Traguriense, dall'altra rivelano interessanti implicazioni sul piano letterario per l'interpretazione dell'intero episodio.

Parole chiave

Petronio, gallina, uova.

Fecha de recepción: 20/04/2010

Fecha de aceptación y versión final: 29/05/2010

La cena era cominciata da poco e si stavano servendo gli antipasti: piatti raffinati, servizio accurato. Encolpio aveva ammirato l'asinello di bronzo corinzio con le due bisacce di olive bianche e nere, i ghiri cosparsi di miele e di semi di papavero e sorretti da una originale struttura, le salsicce su una graticola d'argento, sopra un letto di prugne e grani di melograno a raffigurare le braci. Trimalcione, entrato nel triclinio a cena iniziata, non aveva ancora preso posto. Se ne stava in disparte a concludere, tra le imprecazioni, una partita con uno splendido gioco da tavolo: una tavola in terebinto, legno duro da ebanisteria fine, dadi di cristallo, monete d'oro e d'argento come pedine; la cosa aveva colpito Encolpio. A questo punto arriva nel triclinio un vassoio che regge un canestro con una gallina di legno, accovacciata sulla paglia, con le ali allargate come fanno le chiocce quando covano le uova; si accostano due servi e, mentre parte uno stacco musicale assordante, tirano fuori da sotto la gallina ova pavonina e le distribuiscono ai convitati. Trimalcione solleva un attimo il capo dal gioco e presenta agli ospiti quella portata tutta particolare: "Ho fatto mettere a covare sotto la gallina le uova di pavone, ma ho paura che abbiano già il pulcino: proviamo tuttavia a vedere se si possono consumare" (33.5). Il testo tradito delle parole di Trimalcione presenta però qualche problema e gli studiosi hanno prospettato soluzioni diverse. 
Il codice traguriense H (Parisinus 7989) attesta: temptemus tamen si adhuc sorbilia sunt sorberi possunt; gli excerpta longa dello scaligeriano 1 (Leidensis Scaligeranus 61), le edizioni del Pithoeus $\left(1587^{2}\right.$ ) e del Tornaesius (1575) riportano invece temptemus tamen si adhuc sorbilia sunt. Editori e critici si schierano da subito in due campi: o considerano sorberi possunt semplice glossa esegetica del precedente sorbilia, oppure valorizzano il testo variamente interpungendo e variamente interpretando.

Nicolaus Heinsius, le cui scedae inedite sono confluite con vicende travagliate nelle note di Burman ${ }^{1}$, ritiene che sorberi possunt del traguriense sia da espungere "ut glossa manifestissima". Riferisce però che Th. Muncker accettava il testo del traguriense e considerava tutta la frase "inter stulte dicta Trimalcionis". Sulla scia di Muncker, Burman ${ }^{2}$ propone in nota di leggere sorberique possunt, ma non accoglie la pericope nel testo. Gli editori successivi (Buecheler 1862, Friedlaender 1909², Ernout 1923, K. Müller 1983², Giardina - Cuccioli Melloni 1995) continueranno a considerare una glossa la pericope sorberi possunt del cod. $\mathrm{H}$.

In Italia in particolare si afferma però una tradizione diversa. Marmorale ${ }^{3}$ accoglie esplicitamente la soluzione di Muncker e stampa la pericope così interpungendo: temptemus tamen; si adhuc sorbilia sunt, sorberi possunt. Poco dopo anche un acuto lettore di Petronio, Vincenzo Ciaff, opta per la soluzione conservativa, ma con altra interpunzione, secondo diversa interpretazione: Et mehercules timeo ne iam concepti sint. Temptemus tamen, si adhuc sorbilia sunt. Sorberi possunt ${ }^{4}$; e spiega: “...non si tratta di una freddura del padrone di casa, bensì di una battuta ad effetto teatrale, in cui il si adhuc sorbilia sunt va unito a ciò che precede, e il sorberi possunt, isolato da una pausa cui corrisponde l'assaggio, è la sentenza sullo stato di conservazione o meno delle uova". Anche Paratore difende la lezione sorberi possunt di $\mathrm{H}$; ne deduce però che "Trimalcione in sostanza dice cosa banalissima, sì che ne resta sottolineata la sua grossolana superficialità" ${ }^{6}$. Sulla medesima linea di Muncker e Marmorale anche Castorina ${ }^{7}$ (temptemus tamen;

${ }^{1}$ Così nella praefatio della sua edizione: G.T. Petronius Arbiter, Satyricon, Tomus primus, curante Petro Burmanno, Amstelodami 1743 (= Hildesheim-New York 1974), ristampa rivista da J.J. Reiske dell'edizione di Utrecht 1709.

2 Burman, I, 179.

${ }^{3}$ Petronii Arbitri Cena Trimalcionis. Testo critico e commento a cura di E.V. Marmorale, Firenze 1947, 20.

${ }^{4}$ Satyricon di Petronio, a cura di V. Ciaff, Torino 1967, 116 e traduce: "Ma ho paura, per bacco, che ci sia già la famiglia! Ad ogni modo, proviamo se sono ancora da bere. Sì, si possono bere". Testo e traduzione non variano in Petronio, Satyricon, a cura di V. Ciaffi, Torino 1967, 38-9.

${ }^{5}$ V. Ciaff, Struttura del Satyricon, Torino 1955, 75, n. 8.

${ }^{6}$ E. Paratore, La narrativa latina nell'età di Nerone. La Cena Trimalchionis di Petronio, Roma 1961, 97.

${ }^{7}$ Petronio Arbitro, Dal "Satyricon", introduzione, nota critica, testo e traduzione di E. Castorina, Bologna 1970, 110-1. 
si adhuc sorbilia sunt, sorberi possunt), che però così interpreta: "comunque proviamo: se sono ancora sorbibili, sorbiamoli”. Alla interpretazione di Ciaffi e alla sua "ingegnosa collocazione della punteggiatura" che lascia intuire l'assaggio del padrone di casa, ritorna Aragosti ${ }^{8}$ in una accurata edizione divulgativa che non ignora i problemi dell'opera. Il testo e l'interpretazione di Ciaff, che già si erano rivelati nella discussa traduzione/rivisitazione di Sanguineti ${ }^{9}$, riaffioreranno in altre edizioni divulgative ${ }^{10}$, mentre il testo e la lettura di Muncker ricompariranno anche in edizioni di maggior rilievo ${ }^{11}$.

Risulta evidente che in questo caso la costituzione del testo non può prescindere dalla sua interpretazione: filologia testuale ed esegesi sono reciprocamente vincolate. Riconsideriamo l'episodio. La gallina nel canestro è di legno (gallina... lignea), quindi finta, e da come allarga le ali arrotondate sul corpo, Encolpio, il narratore, capisce che rappresenta una chioccia che sta covando le uova (patentibus in orbem alis, quales esse solent quae incubant ova).

A questa gallina di legno sono state talora accostate, con funzione esegetica, altre galline, vere, ricordate da Giovenale (11.70):

grandia praeterea tortoque calentia faeno ova adsunt ipsis cum matribus...

ci sono inoltre grosse uova, calde nel fieno arrotondato, con le stesse galline.

Sulla scorta di questa attestazione di Giovenale si è arrivati a immaginare quasi l'uso romano di portare direttamente nel triclinio le galline con il nido dove depongono le uova, a garantire ed esaltare la freschezza del loro prodotto ${ }^{12}$. Per la verità anche il capretto che apre la lista delle vivande di Giovenale potrebbe vantare diritto di presenza in vita nel triclinio; più problema-

\footnotetext{
${ }^{8}$ Petronio, Satyricon, introduzione, traduzione e note di A. Aragosti, BUR, (Milano) 1995, 202, n.72.

${ }^{9}$ E. Sanguineti, Il giuoco del Satyricon: un’imitazione da Petronio, Torino 1970, 40 (= Satyricon di Petronio, nella traduzione di E. S., Torino 1993, 36): "Ma insomma, che noi ci proviamo un po', che poi magari sono ancora buone da bere. E ma sì, guarda, che si possono anche bere davvero".

${ }^{10}$ Petronio, Satyricon, a cura di Mariangela Scarsi, Milano 2004, 56-7: Temptemus tamen, si adhuc sorbilia sunt. Sorberi possunt. "Proviamo comunque se si possono ancora succhiare. Sì, si possono succhiare".

${ }^{11}$ Petronius, Cena Trimalchionis, A New Critical Edition by J. Öberg, Studia Latina Stockholmiensia XLII, Stockholm 1999, 33: Temptemus tamen: si adhuc sorbilia sunt, sorberi possunt. E precisa in nota che il passo va inteso "inter stulte dicta Trimalchionis", ritornando così all'interpretazione di Muncker.

12 "Era dunque un uso comune, quasi a dimostrar la freschezza delle uova": così Marmorale p. 20 commenta i due versi di Giovenale, totalmente decontestualizzati. Sulla scorta del medesimo passo (Iuv. 11.70) E. Courtney, A Companion to Petronius, Oxford-New York 2001, 83 osserva: “... hen eggs ... were sometimes presented at table in this manner".
} 
tico con le verdure. Giovenale in realtà nella satira undicesima parla d'altro e altro testimonia; certamente non prospetta la presenza di vivande vive nel triclinio. Il poeta sta infatti deprecando le spese folli per cibi esotici, rovinose per le finanze personali, e vi contrappone una cena costituita di portate che non necessitano di acquisti al mercato: Fercula nunc audi nullis ornata macellis (11.64); enumera un capretto da latte, asparagi di monte, uova freschissime e galline, uva, pere e mele; conclude che questa era già una cena lussuosa per i senatori di un tempo (11.65-78). Giovenale non attesta dunque la presenza, oltre tutto operativamente problematica, di galline nel nido con uova fresche nei triclini romani, ma semplicemente dichiara una disponibilità di uova fresche e galline ${ }^{13}$; e la gallina sezionata ritorna al v. 135 non... peior gallina secatur; offerta di uova e pollo, cibi semplici, all'ospite, anche nella Cena petroniana: Echione, uno dei liberti, rivolto ad Agamennone: aliqua die te persuadeam ut ad villam venias et videas casulas nostras. Inveniemus quod manducemus, pullum, ova: belle erit, etiam si omnia hoc anno tempestas depravavit: inveniemus ergo unde saturi fiamus. (Petron. 46.2). Uova e pollame, ancora come vivande di una cena, sono ricordati assieme più volte anche da Orazio e Marziale ${ }^{14}$.

Proprio Marziale ricorda in particolare, come Giovenale, ova matrum, uova di gallina ${ }^{15}$, in un catalogo di cibi rustici e semplici destinati a un amico (7.31.1): e matres non sono le chiocce o le madri, come talora si intende ${ }^{16} ; \mathrm{ma}$ -

${ }^{13}$ Lo scolio a Iuv. 11.70 è chiaro: recentia ova cum ipsis gallinis (Scholia in Iuvenalem vetustiora, collegit recensuit illustravit P. Wessner, Lipsiae 1931, 186); e tortoque... faeno indica il fieno arrotondato a circolo, a costituire il nido; per la iunctura vd. Prop. 4.2.25 da falcem et torto frontem mihi comprime faeno, già riecheggiato da Ov. met. 14.643 tempora saepe gerens faeno religata recenti; ma il modello è virgiliano georg. 1.349 torta redimitus tempora quercu; il fieno serviva anche per il trasporto delle uova Mart. 3.47.13 tuta faeno cursor ova portabat.

${ }^{14}$ Mart. 3.58.50 holus, ova, pullos, poma...; 7.31.1 Raucae chortis aves et ova matrum; 7.54.7 non chortis aves, non ova supersunt; 11.52.8-9 quam... ova tegant; / altera non derunt tenui versata favilla; 14 et chortis saturas atque paludis aves; e già in Hor. ep. 2.2.163 uvam, pullos, ova; e in sat. 2.2.121-2 ...pullo atque haedo;... uva / nux ... duplici ficu.

${ }^{15}$ Più esattamente nel contesto dovrebbe indicare più in generale "volatili da cortile".

16 'Chioccia' traduce Ceronetti (M.V. Marziale, Epigrammi, versione di G. Ceronetti, Torino 1964², 443), 'chiocce' intende Scandola in Marziale, Epigrammi, Saggio introduttivo di Mario Citroni, traduzione di Mario Scandola, note di Elena Merli, Milano 1996. Guillermo Galán Vioque, Martial, Book VII; A Commentary, Leiden-Boston-Köln 2002, 220, riferisce matrum a aves: ova matrum: ie, ova earum (chortis avium) matrum; già la vecchia edizione Pomba (Marci Valeri Martialis Epigrammata, Torino 1883, I, 628) interpretava, più puntualmente: matrum: gallinarum, anatum. Vd. anche la testimonianza di Ov. Fast. 4.696 matris plumis ova fovenda dabat. Per indicare l'equivalente della nostra chioccia, Columella e Palladio attestano il sostantivo matrix, sia per le galline che per altri volatili, termine usato da Varrone e Columella anche per indicare le fattrici dei mammiferi (bovini, ovini, suini...); ma il loro rapporto con i piccoli (pulli) è sempre definito da mater; paradigmatico Pallad. 12.13.1 ac primo per biduum natus cum matre claudatur, tunc septis obscuris servetur et calidis; ita secluso parvulorum grege, matrices mittantur in pascua: la pecora è mater finchè sta con l'agnello, matrix quando è con le altre fattrici. 
tres indica, qui come nel passo di Giovenale, galline mature, ovaiole, dal momento che per i latini la deposizione dell'uovo è pur sempre un partorire ${ }^{17}$.

Le testimonianze di Marziale e Giovenale attestano quindi solo l'uso culinario di uova e galline e non possono dunque essere invocate per spiegare la scena della gallina di Trimalcione: per questo dobbiamo ritornare al testo di Petronio.

Alcuni elementi sono chiari e indiscutibili. La gallina è di legno ed è rappresentata nell'atto di covare, è cioè una chioccia; le uova di pavone, in ogni caso segno di un lusso raffinato ${ }^{18}$ e prodotto di una cucina elaborata, sono ugualmente artefatte e i convitati se ne rendono conto appena le prendono in mano (ovaque ex farina pingui figurata pertundimus): nessuno dunque cade nell'equivoco, impossibile, di ritenerle autentiche uova di pavone, veramente covate ${ }^{19}$. Rimane però una curiosità: perché mai Trimalcione annuncia di servire ai convitati uova di pavone già poste a covare sotto la gallina? Si sa che chiocce e uova fresche sono incompatibili. Ma perché Trimalcione avrebbe dovuto servire delle uova ripiene presentandole come già in parte covate, correndo il rischio di rovinare la presentazione della portata raffinata? È una stramberia senza senso di Trimalcione, che però poco dopo dirà del suo operare nihil sine ratione facio (39), oppure il tutto può avere una sua intrinseca spiegazione? Il problema diventa tecnico, connesso al procedimento per la riproduzione dei pavoni.

Per una economia rurale, secondo la testimonianza di Columella in particolare, la femmina di pavone risulta molto più redditizia per la produzione di uova $^{20}$ che per le cure parentali, tanto più che il maschio la disturba durante la cova, contribuendo a sciupare qualche uovo ${ }^{21}$. La soluzione escogitata dagli allevatori antichi, e proseguita dai moderni, consiste nel far covare le uova di pavone alle galline, chiocce decisamente più volonterose. Per questo è del

${ }^{17}$ Concetto confermato per la deposizione delle uova degli ovipari dall'uso del lessico relativo alla generazione: più frequente pario / parturio (ThlL s. u. pario 10.1, 406.49-407.11 Kruse), accanto al più raro edo (ThlL 5.2, 84.47 ss. Kapp-Meyer); in questa ottica, Palladio (1.28.3) definisce nutrices le gallinae vicarianti che covano uova di pavone.

${ }^{18}$ A definire l'ambiente sociale cui rinvia l'immagine del pavone, merita ricordare le parole con cui Columella apre il capitolo relativo all'allevamento: Pavonum educatio magis patrisfamilias quam tetrici rustici curam poscit (8.11.1).

${ }_{19}$ Per aspetti rilevanti della cucina e del triclinio di Trimalcione, come la manipolazione degli alimenti e l'illusionismo culinario, qui non considerati, vd. G. Rosati, Trimalcione in scena, Maia 35, 1983, 213-27: 221ss. = Trimalchio on Stage, in Oxford Readings in the Roman Novel, ed. by S. J. Harrison, Oxford-New York 1999, 85-104: 96ss.

${ }^{20}$ Colum. 8.11.9-10 ova ... quae quanto recentiora gallinis subiecta sunt, tanto commodius excuduntur, idque fieri maxime patrisfamiliae ration $<i>$ conducit. Nam feminae pavones quae non incubant ter anno fere partus edunt, at quae fovent ova totum tempus fecunditatis aut excudendis aut etiam educandis pullis consumunt.

${ }^{21}$ Plin. nat. 10.161 mares ea (scil. ova) frangunt desiderio incubantium; Pallad. 1.28.2 masculi ova et pullos suos persequuntur velut alienigenas; ma la notazione è già in Aristot. H.A. 564b.2-5. 
tutto normale che Trimalcione collocasse le uova di pavone sotto la gallina di legno: rifletteva una pratica diffusa, come ci informano gli autori che si occupano di res rusticae ${ }^{22}$. Assodato che la presentazione trova corrispondenza nel procedimento reale, rimane però intatto l'interrogativo di partenza: per quale ragione Trimalcione pensa di presentare agli ospiti le uova ripiene come uova già covate? Come si giustifica tale presentazione?

La risposta, crediamo, è correlata ancora una volta al procedimento riproduttivo dei pavoni, attestato dalle fonti e descritto in forma dettagliata da Columella ${ }^{23}$. Le uova di pavone si schiudono dopo una cova di ventisette/ trenta giorni; quelle di gallina invece dopo venti/ventuno. Ma la gallina, che cova assieme uova di pavone e di gallina, non potrebbe continuare a covare le uova di pavone fino al ventottesimo giorno e insieme accudire ai suoi pulcini nati al ventunesimo giorno: per l'allevatore sarebbe un disastro. L'allevatore, ci informano sempre gli scrittori di res rusticae, dovrà ovviare all'inconveniente in questo modo: metterà a covare sotto una gallina un certo numero di uova di pavone assieme a uova di gallina (in modo da far accettare alla gallina le uova di pavone); dopo una decina di giorni sostituirà le uova di gallina già parzialmente covate con altrettante fresche di gallina: in questo modo sia quelle di gallina che quelle di pavone si schiuderanno assieme al ventisettesimo/trentesimo giorno di cova e la chioccia si prenderà cura di tutti i pulcini. Al di là della felice riuscita della covata, a noi interessa rilevare un elemento: chi mette a covare uova di pavone sotto una chioccia di gallina, secondo il metodo illustrato da Columella e Varrone, si trova ad avere a disposizione delle uova parzialmente covate, delle quale potrebbe dire, proprio come Trimalcione: timeo ne iam concepti sint. La loro consumazione doveva comportare qualche rischio. Con ogni probabilità è da questa situazione che trae origine l'idea di Trimalcione di presentare uova ripiene particolarmente elaborate come se fossero uova già in parte covate e forse non più consumabili.

Si potrà obiettare che si tratta di una informazione tecnica piuttosto sofisticata, molto particolare, forse di non comune conoscenza. Queste conoscenze certamente in una società agreste erano più diffuse di quanto non lo siano nella società moderna, dove i metodi di produzione sono spesso noti solo agli addetti ai lavori. Ma il fatto stesso che Trimalcione ponga le sue uova di pavone sotto una gallina, dimostra che il metodo è noto. Anche un autore come Cicerone, in un'opera insospettata, ci dà testimonianza di tali conoscenze.

${ }^{22}$ Colum. 8.11.9 e più sinteticamente Varro, rust. 3.9.10 e Plin. nat. 10.161; Smith riporta nel suo commento le informazioni varroniane (Petronii Arbitri Cena Trimalchionis, ed. by M.S. Smith, Oxford 1975, 71) e W.T. Avery, in una rapida nota (More Petroniana, Hermes $107,1979,118-21)$, riferisce le testimonianze di altri autori, ma nessun studioso pone il problema dell'insolito modo di servire le uova di pavone.

${ }^{23}$ Varro, rust. 3.9.10; Colum. 8.11.9; Pallad. 1.28.3. Raccoglie le fonti antiche relative al pavone F. Capponi, Ornithologia Latina, Genova 1979, 389-93. 
Afferma Cicerone nel de natura deorum che ogni specie ha una sua natura e adduce tra le altre prove il fatto che dalle uova di anatra covate dalle galline nascono anatre con tutte le peculiarità della specie ${ }^{24}$ : simili tecniche erano dunque note a Cicerone stesso e certamente diffuse se le offre come prova ai lettori di una sua opera filosofica.

Dunque tutto chiaro? Non proprio. L'allevatore che sostituisce le uova di gallina covate con con quelle di gallina non ancora covate si trova con uova di gallina in parte già covate; ma sono, per l'appunto, uova di gallina; Trimalcione invece presenta come già covate le uova di pavone ${ }^{25}$. E' ovviamente da escludere un errore dell'autore, cioè di Petronio: la presenza della gallina e delle uova di pavone garantiscono la conoscenza del metodo di riproduzione. L'imprecisione è dunque imputabile al personaggio Trimalcione, che sbaglia uova: sarebbe una delle sue numerose approssimazioni. E qui emerge la funzione dell'autore 'nascosto' che gioca col suo personaggio, esattamente come gioca con Encolpio, l'io narrante ${ }^{26}$. Encolpio vede la gallina di legno, riceve le uova ripiene confezionate con farina grassa, simili a uova di pavone, ma quando intravede quello che in realtà si rivelerà poi un grasso beccafico, per poco non getta via tutto, quasi si trattasse veramente di un uovo con il pulcino (ego quidem paene proieci partem meam, nam videbatur mihi iam in pullun coisse 33.7). Eppure gli era chiarissimo fin dall'inizio che tutto era finto. Anzi proprio la presenza di uova di pavone risulta tranquilizzante: non c’è motivo per sottrarre alla cova le uova di pavone, mentre, eventualmente, proprio quelle di gallina, quelle sostituite dopo dieci giorni, avrebbero potuto ingenerare qualche sospetto.

Dunque tutto è patentemente finto, tranne il beccafico nel tuorlo pepato. Ma Trimalcione non voleva ingannare proprio nessuno, anzi con quel servizio, con quelle guarnizioni non avrebbe potuto ingannare nessuno neppure se l'avesse voluto. Le uova di pavone, e non di gallina, ne sono un ulteriore segno.

L'idea delle (finte) uova di pavone covate dalla gallina di legno proviene dunque dalla realtà, riprodotta nell'apparato della mensa; allo stesso modo in precedenza le olive erano contenute nelle bisacce dell'asinello di bronzo perché così nella realtà l'asino trasportava le olive; e i ghiri erano sorretti e vivacizzati da ponticuli ferruminati e le braci, rappresentate da prugne nere e grani rossi di melograno, arrostivano le salsicce su una graticola

${ }^{24}$ Cic. nat. 2.124 quin etiam anitum ova gallinis saepe subponimus; e quibus pulli orti primo aluntur ab his ut a matribus, a quibus exclusi fotique sunt;

${ }^{25}$ Le uova di pavone sono più grosse di quelle di gallina e a Trimalcione, o meglio al suo cuoco, servono uova grosse proprio perché devono contenere il beccafico nel tuorlo d'uovo pepato.

${ }^{26}$ Il riferimento è ovviamente all'analisi di G.B. Conte, The hidden author: an interpretation of Petronius' Satyricon, Berkeley 1996 = L'autore nascosto. Un'interpretazione del Satyricon, Bologna 1997 (Pisa 2007²). 
d'argento: così la gallina di legno ricorda le galline vere, con le loro covate di ova pavonina. Trimalcione dunque riproduce nella contraffazione una realtà apparentemente degradata (chioccia e uova con pulcino), confidando e puntando sulla immediata verifica dei convitati, sulla scoperta del beccafico, che scioglie ogni tensione. Potremmo dire, paradossalmente, non che le uova sono collocate sotto la gallina perché contengono il finto pulcino, ma hanno il finto pulcino perché nella realtà, quelle vere, potevano provenire da sotto la gallina con un pulcino vero.

La trovata di Trimalcione è tutta nell'apparato del servizio, nel modo di presentare quelle uova di pavone abilmente confezionate col loro ripieno, nel tentativo di provocare nei commensali un momentaneo imbarazzo che immediatamente però doveva apparire ingiustificato agli ospiti, come in effetti avviene. Ma con Encolpio, ospite raffinato, il gioco non funziona; anzi, con lui la trovata spiritosa di Trimalcione ottiene l'effetto opposto; la realtà disvelata del beccafico, pur nell'uovo artefatto, genera reale disgusto (ego quidem paene proieci partem meam 33.7) proprio per le parole di presentazione (et mehercules timeo ne iam concepti sint 33.5) e ci vuole la persuasione del commensale vicino (hic nescio quid boni debet esse 33.8) per convincere Encolpio a continuare ad aprire il guscio dell'uovo (persecutus putamen manu 33.8). La sua reazione non rivela dunque né ingenuità né accondiscendenza $^{27}$; lascia invece intuire il giudizio di Petronio, un giudizio garbato ma senza appello, sui gusti e sulle conoscenze approssimate di Trimalcione.

Se questa è la situazione della scaen $a^{28}$ della gallina con le uova di pavone, qualche logica conseguenza ne deriva anche per l'incertezza testuale prospettata all'inizio. In una performance così accuratamente preparata, sia in cucina sia nel servizio in triclinio, le parole di Trimalcione secondo il testo (si adhuc sorbilia sunt, sorberi possunt) e l'interpretazione di Muncker ("inter stulte dicta Trimalcionis"), finiscono per sciupare tutto il gioco e risultano inoltre inconciliabili con le premesse della scena, dove tutto è manifestamente finto. Dall'altra parte la fine soluzione individuata da Ciaffi non risulta proponibile, perché presuppone che Trimalcione finga di invogliare e ingannare con l'assaggio i commensali: temptemus tamen si adhuc sorbilia sunt. Sorberi possunt $(\mathrm{H})$. Trimalcione svolgerebbe opera di convinzione per rendere più forte, per esasperare l'equivoco all'apertura delle uova. Ma,

${ }^{27}$ A un Encolpio ingenuo pensa C. Panayotakis, Theatrum Arbitri. Theatrical elements in the Satyrica of Petronius, Leiden-New York-Köln 1995, 68; una finta ingenuità per ingraziarsi Trimalcione prospetta N.W. Slater, Reading Petronius, Baltimore-London 1990, 59 n. 20.

${ }^{28}$ Per la presenza di elementi teatrali nella Cena, anche per questa scena, vd. G. Rosati, "Trimalcione in scena", Maia 35, 1983, 213-27: 215, 219-20 = Trimalchio on Stage, in Oxford Readings in the Roman Novel, ed. by S. J. Harrison, Oxford-New York 1999, 85-104: 87-8, 94-5; C. Panayotakis, Theatrum Arbitri. Theatrical elements in the Satyrica of Petronius, Leiden-New York-Köln 1995, 67-8. 
come si è visto, tutto è dichiaratamente finto e, soprattutto, Trimalcione sta ancora giocando con la tabula lusoria, e si limita a volgere il capo verso il servizio del triclinio (convertit ad hanc scenam Trimalchio vultum... 33.5); non è quindi nelle condizioni di poter fungere da garante, quasi avesse controllato e assaggiato di persona. Tanto più (e questo ne è la riprova) che solo successivamente, messo da parte il gioco, si farà portare quanto era già stato servito agli altri: iam Triamalchio eadem omnia, luso intermisso, poposcerat...34.1. Il contesto dunque induce a ritenere, inequivocabilmente, sorberi possunt $(\mathrm{H})$ glossa del precedente sorbilia, un termine tecnico e raro, direi specialistico, che giustifica la glossa stessa ${ }^{29}$.

Tutto è dunque patentemente finto in questa scena, tranne il beccafico, il tuorlo d'uovo pepato e lo spirito schiettamente popolano e un po' furbesco di Trimalcione, che però non supera indenne il giudizio di Petronio, maestro riconosciuto di raffinato buon gusto.

${ }^{29}$ L'attributo sorbilis è molto meno comune di quanto possa sembrare. Se escludiamo il caso di Columella 8,17 (cibi sorbilibus proximi, ut e mulctra recens caseus, si loci conditio vel lactis annona permittit), e di Cassio Felice de med. 46 (si vero sitis minime fuerit, non frigidis sed humidis tantum cibis nutries, id est sorbilibus multa aqua solutis, ut sunt ptisanae, ova sorbilia, micae panis diligenter elutae et cetera quae in sorbitionibus similiter accipiuntur), in tutte le altre occorenze l'attributo è sempre esplicitamente riferito all'uovo; non solo, ma ricorre sempre e solo in opere di medicina, da Celso (I sec. d.C.; 8 attestazioni) a Scribonio Largo (I sec. d.C.; 1 occorrenza), da Marcello Empirico (V sec.; 9 casi) a Cassio Felice (V sec.; 2 casi), da Anthimo (VI sec.; 3 casi), a Sorano/Mustio (VI d.C.?; 2 casi) alla Medicina Plinii (III sec.; 1 caso): dunque è voce tecnica del lessico medico (ambito in cui Trimalcione dimostrerà una certa competenza) e questo può spiegare l'origine della glossa, facilitata forse dalla presenza ricorrente nei testi grammaticali della forma verbale sorbillo da sorbeo, come esempio di derivazione cum deminutione. 
